\title{
La influencia de la actividad física como tratamiento alternativo a la prostatitis crónica: un metaanálisis
}

\author{
Juan Pablo Hervás-Pérez ${ }^{a, *}$, Victor Jiménez Díaz-Benito ${ }^{\mathrm{b}}$, \\ Jesús Guodemar-Pérez ${ }^{\mathrm{a}}$, Montserrat Ruiz-López ${ }^{\mathrm{c}}$, Pablo García-Fernández ${ }^{\mathrm{d}}$, \\ Elena Sonsoles Rodríguez-López ${ }^{\mathrm{a}}$ y David Pérez-Manchón ${ }^{c}$
}

\footnotetext{
a Departamento de Fisioterapia, Facultad de Educación y Salud, Universidad Camilo José Cela, Villanueva de la Cañada, Madrid, España

b Facultad de Ciencias de la Actividad Física y del Deporte, Universidad Politécnica de Madrid, Madrid, España

c Departamento de Enfermería, Facultad de Educación y Salud, Universidad Camilo José Cela, Villanueva de la Cañada, Madrid, España

'Departamento de Fisioterapia, Facultad de Ciencias de la Salud, Universidad Alfonso X, Villanueva de la Cañada, Madrid, España
}

Recibido el 27 de julio de 2018; aceptado el 2 de diciembre de 2018

Disponible en Internet el 11 de marzo de 2019

\section{PALABRAS CLAVE \\ Prostatitis crónica; Dolor pélvico; \\ Deporte; \\ Atletas; \\ Actividad física}

\begin{abstract}
Resumen
Introducción: La prostatitis crónica es uno de los diagnósticos más comunes en las consultas ambulatorias de Urología. Se presenta como un conjunto heterogéneo de enfermedades, cuya etiología muchas veces resulta desconocida, mostrando como denominador común un conjunto de síntomas dolorosos, irritativos y obstructivos del tracto genitourinario y el periné.

Objetivos: Se realizó una revisión sistemática y metaanálisis actualizado de esta dolencia en relación con los perjuicios o beneficios que la actividad física podría tener en la prostatitis crónica o el dolor pélvico crónico.

Métodos: Se realizó una búsqueda formal en las bases de datos Scopus, PubMed, ScienceDirect, PEDro, The Cochrane Library, Dialnet y SciELO hasta junio de 2018 (siendo el último artículo empleado en este metaanálisis publicado en marzo de 2018).

Resultados: El equipo investigador revisó un total de 93 estudios, de los cuales se seleccionaron 10 , con un posterior examen de su calidad metodológica mediante la escala PEDro. Se realizó la comparación del índice de masa corporal, la calidad de vida relacionada con el índice de masa corporal y la correlación de la incontinencia urinaria. El análisis global de las intervenciones dentro de la incontinencia urinaria resultó significativo (tamaño del efecto: 0,11 ; IC 95\% 0,038 a 0,$43 ; p=0,024)$.
\end{abstract}

* Autor para correspondencia.

Correo electrónico: jphervas@ucjc.edu (J.P. Hervás-Pérez). 
Conclusiones: La variabilidad asociada a diseños experimentales representa una heterogeneidad en los efectos de los distintos programas o intervenciones de actividad física para el tratamiento de la prostatitis crónica. Los tamaños del efecto obtenidos evidencian que la efectividad de los programas de tratamiento alternativos, empleando vehicularmente la actividad física, puede guardar una relación con el tipo de intervención realizada.

(c) 2019 Asociación Española de Andrología, Medicina Sexual y Reproductiva. Publicado por Elsevier España, S.L.U. Todos los derechos reservados.

\section{KEYWORDS}

Chronic prostatitis;

Pelvic pain;

Sport;

Athletes;

Physical activity

\section{Introducción}

La prostatitis crónica/síndrome del dolor pélvico crónico (CP/CPPS) es uno de los diagnósticos más comunes en las consultas ambulatorias de Urología, con una elevada prevalencia entre los varones mayores de 40 años $^{1}$. En vista de la condición multifactorial del CP/CPPS, la literatura científica no ha establecido un consenso que aborde una definición global y aceptada internacionalmente del propio término. Se trata, por tanto, de un conjunto heterogéneo de enfermedades, de causa muchas veces desconocida (9095\% de los casos), aunque sí está bien definida en el resto de los casos la etiología bacteriana (5-10\% de los casos) $)^{2-4}$. Los síntomas que provoca (infección urinaria, disuria, alta frecuencia miccional) resultan dolorosos, irritativos y obstructivos referidos al tracto genitourinario y al periné del varón, acompañados o no de disfunción sexual ${ }^{2,4}$.

El National Institute of Diabetes and Digestive and Kidney Diseases/National Institute of Health clasifica la CP/CPPS como una prostatitis de categoría III (tabla 1) $)^{5}$. Estos
Tabla 1 Clasificación de prostatitis según el National Institute of Diabetes and Digestive and Kidney Diseases/National Institute of Health

\begin{tabular}{ll}
\hline II & Prostatitis aguda bacteriana \\
III & Prostatitis crónica bacteriana \\
III A & CP/CPPS \\
& CPPS inflamatorio: leucocitos en \\
& secreción prostática \\
& espontánea/orina posmasaje \\
& prostático (VB3)/semen \\
& CPPS no inflamatorio: no \\
III B & leucocitos en secreción prostática \\
& espontánea/orina posmasaje \\
& prostático (VB3)/semen \\
& Prostatitis inflamatoria \\
& asintomática (prostatitis \\
IV & histológica) \\
\hline
\end{tabular}

CP: prostatitis crónica; CPPS: síndrome del dolor pélvico crónico. 
pacientes usualmente ven afectada de modo sustancial su calidad de vida.

Existen numerosos artículos que relacionan la práctica de determinados deportes con la aparición de prostatitis ${ }^{6-10}$, sin embargo, para contrarrestar esta visión, la revisión que aquí proponemos trata de mostrar cómo se puede utilizar la actividad física a modo de alternativa terapéutica.

En 2016 una revisión analizó las alternativas terapéuticas existentes, encontrado diversas terapias para el tratamiento de la CP/CPPS, como modificaciones en los estilos de vida, fitoterapia, extractos bacterianos orales, inmunoestimulantes, acupuntura, terapia miofascial y biofeedback, terapias neuromoduladoras, ondas de choque extracorpóreas, fármacos con efecto sobre el sistema central y periférico, terapias cognitivo-conductuales y terapias térmicas ${ }^{3}$. Estudios transversales y longitudinales muestran que cantidades crecientes de actividad física están asociadas con una disminución de la mortalidad y una mejor salud y funcionamiento, incluida una disminución de los síntomas de ansiedad, estrés y depresión ${ }^{11,12}$.

Presentamos una revisión sistemática actualizada de esta dolencia analizando los perjuicios o beneficios que la actividad física podría tener en la CP/CPPS.

\section{Metodología}

\section{Adquisición de la evidencia}

La revisión sistemática se llevó a cabo siguiendo los criterios del Preferred Reporting Items for Systematic Reviews and Meta-Analyses ?PRISMA?, que tiene como objetivo mejorar el estándar de presentación de informes de revisiones sistemáticas y metaanálisis ${ }^{13}$. Para realizar este trabajo se realizó una revisión de la evidencia publicada hasta junio del 2018 empleando los términos científicos: prostatitis, chronic pelvic pain syndrome, sport, athletes y physical activity, combinados con los operadores booleanos (AND; OR). Las bases de datos utilizadas fueron Scopus, PubMed, ScienceDirect, Physiotherapy Evidence Database (PEDro), The Cochrane Library, Dialnet y SciELO. Todas las búsquedas fueron realizadas por 2 investigadores independientes. Se incluyeron artículos publicados hasta junio del 2018 en español/inglés. Los criterios de exclusión fueron literatura no española/inglesa o artículos con graves defectos metodológicos.

\section{Evaluación de la calidad metodológica}

Esta evaluación de la calidad metodológica (validez externa, validez interna e informes estadísticos) se efectuó a través de la escala PEDro ${ }^{14,15}$ (demostrada herramienta válida y fiable para este fin), mientras que el nivel de evidencia se estableció de acuerdo con la escala del Oxford Centre for Evidence-Based Medicine ${ }^{16}$. Ambos parámetros se evaluaron de forma independiente por 2 de los autores y todos los desacuerdos surgidos se resolvieron hasta alcanzar el consenso.

\section{Análisis cuantitativo}

Los datos fueron registrados en una base de datos de Excel (versión 2013 para Windows 10) según la información sobre la población, las características del estudio y las medidas de resultados. Para cada medida de interés se estimó un tamaño del efecto (TE) sobre los datos originales del artículo revisado ${ }^{17}$ por Hedges y Larry a través del software Effect Size Determination Program ${ }^{18}$. Para ello se utilizaron las informaciones obtenidas de las medias, las desviaciones típicas, las pruebas t de los diferentes estudios y otros estadísticos de contraste como la F de Snedecor. Para corregir el sesgo positivo de la diferencia media tipificada para tamaños muestrales pequeños, se corrigió la d resultante ${ }^{17}$. Se adoptó el modelo de efectos fijos, que determina la homogeneidad de las medidas de tratamiento de los diferentes estudios. La homogeneidad de los estudios se contrastó mediante el procedimiento propuesto por Hedges y Olkin que implica calcular el estadístico $Q$, el cual se distribuye según Chi cuadrado con $\mathrm{k}^{-1}$ grados de libertad, donde $\mathrm{k}$ es el número de $\mathrm{TE}^{19}$. Entre los diferentes estudios, se presenta el TE, ya corregido, junto con el intervalo correspondiente, para un intervalo de confianza (IC) de un $95 \%$. Se consideró un TE pequeño, cuando el estadístico para una puntuación se sitúa alrededor de 0,2; alrededor de 0,5 representó un efecto medio y en torno a 0,80 un efecto grande ${ }^{20}$. Las diferencias significativas fueron consideradas viendo que el TE de una intervención no se contempló dentro de un IC de un $95 \%$.

\section{Resultados}

\section{Síntesis de la evidencia}

De los 93 artículos encontrados inicialmente en la revisión durante los últimos 10 años, 27 fueron descartados por aparecer duplicados, 15 se suprimieron por ser cartas al director, notas, abstracts o presentaciones en congresos. Además, se descartaron 5 artículos por el idioma, al no estar redactados en inglés o castellano. Por último, 6 más fueron eliminados por tener una temática diferente a la prostatitis en el deporte. Se seleccionaron finalmente 10 artículos que cumplieron los criterios de inclusión, tal y como se muestra en el diagrama de flujo tipo CONSORT (fig. 1).

\section{Evaluación de la calidad metodológica}

Se analizaron metodológicamente los 10 estudios que cumplían los criterios de inclusión. Con el objetivo de valorar la calidad metodológica de los estudios seleccionados, se les aplicó la escala PEDro, la cual presenta 11 ítems de respuesta «sí», «no» o «no informa», de los cuales solo 10 son evaluables, ya que el primero no se puntúa al referirse a la validez externa del estudio. Los ítems contestados con «sí» reciben un punto; el resto, no recibe ninguno. Autores como Moseley et al. defienden que una puntuación igual o mayor de 5 denota una buena calidad metodológica y un riesgo de sesgo bajo ${ }^{21}$. Se observa una calidad metodológica de buena a excelente, puntuando todos entre 5 y 10 puntos sobre 10 , con una media de $6,00 \pm 1,15$ (tabla 2 ). 


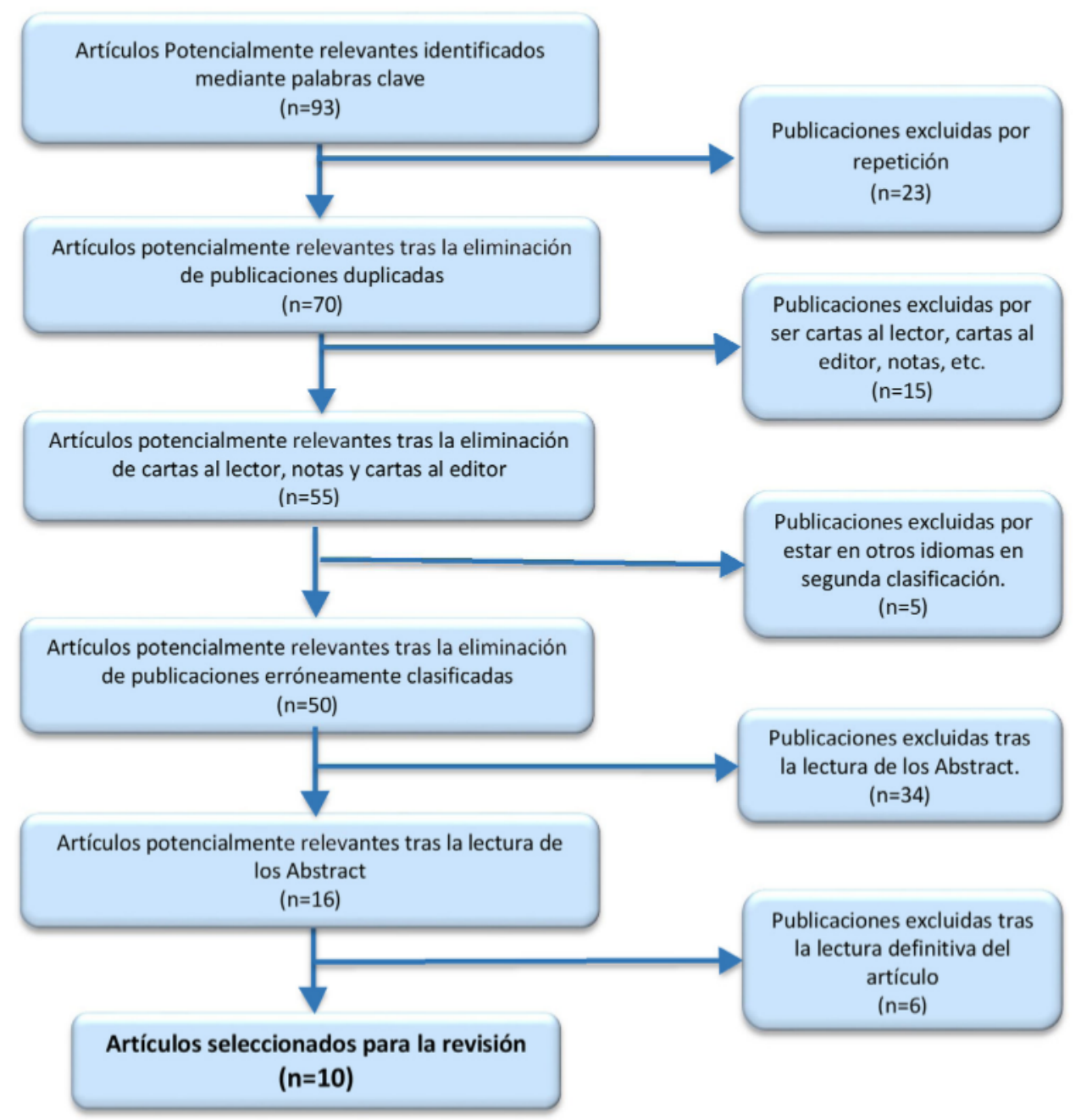

Figura 1 Diagrama de flujo explicativo de la revisión bibliográfica.

\section{Extracción y análisis de datos}

Se realizó una evaluación de los estudios seleccionados por 2 revisores de manera independiente, extrayéndose a una tabla los siguientes datos: autor, año de publicación del artículo, protocolo de intervención, tamaño de la muestra, tipo de estudio, duración, seguimiento y resultados obtenidos.

\section{Síntesis de los resultados}

De los estudios revisados, se recogieron 7 estudios observacionales que recogen relaciones entre los síntomas CP/CPPS y la actividad física y 3 estudios donde a través de un programa específico de ejercicios evalúan los síntomas asociados con CP/CPPS. En la tabla 3 se resumen los datos significativos obtenidos de los artículos estudiados.

\section{Estimaciones de efectos combinados de las medidas de resultado}

Referente al TE para poder realizar las estimaciones válidas para el modelo de metaanálisis, se realizó la comparación del índice de masa corporal, la calidad de vida relacionada con el índice de masa corporal y la comparación de la incontinencia urinaria (figs. 2-4).

Dentro del análisis de la comparación del índice de masa corporal, se analizaron 4 comparaciones de forma independiente, con una muestra de 8.896 sujetos (fig. 2). No se hallaron diferencias significativas en el índice de masa corporal con síntomas urinarios $(p>0,05)$. De las 4 intervenciones estudiadas, el análisis de todas las intervenciones desde un punto de vista global mostró que los TE no tuvieron un efecto significativo (TE: 0,033; IC 95\% -0,038 a 0,092; $p=0.4557$ ).

Cuando se analiza la calidad de vida relacionada con el índice de masa corporal, se estudiaron 2 comparaciones de forma independiente, con una muestra de 442 sujetos (fig. 3). No se hallaron diferencias significativas en la calidad de vida con síntomas urinarios $(p>0,05)$. De forma global, el análisis no mostró un TE significativo (TE: 0,025 ; IC $95 \%$ $-0,038$ a 0,092; $p=0,4557)$.

Por último, se analizaron 2 comparaciones de forma independiente con relación a la incontinencia urinaria, con una muestra de 483 sujetos (fig. 4). No se hallaron diferencias significativas en incontinencia urinaria $(p>0,05)$. El análisis 
Tabla 2 Resultados de la escala PEDro

\begin{tabular}{|c|c|c|c|c|c|c|c|c|c|c|c|c|c|c|}
\hline \multirow[t]{2}{*}{ Referencia } & \multirow[t]{2}{*}{ Tipo de estudio } & \multicolumn{12}{|c|}{ PEDro } & \multirow{2}{*}{$\begin{array}{l}\text { Conflicto de } \\
\text { intereses }\end{array}$} \\
\hline & & $\mathrm{E}$ & 2 & 3 & 4 & 5 & 6 & 7 & 8 & 9 & 10 & 11 & Total & \\
\hline Giubilei et al.22, 2007 & Estudio aleatorizado & + & + & + & + & + & + & - & + & + & + & + & 9 & - \\
\hline Bartoletti et al. ${ }^{23}, 2009$ & $\begin{array}{l}\text { Estudio observacional } \\
\text { analítico de cohorte }\end{array}$ & + & - & - & + & + & - & - & + & - & + & + & 5 & - \\
\hline Serdà et al. ${ }^{24}, 2010$ & $\begin{array}{l}\text { Estudio } \\
\text { cuasiexperimental }\end{array}$ & + & + & - & + & - & - & - & + & + & + & + & 6 & No \\
\hline Serdà et al. ${ }^{25}, 2013$ & $\begin{array}{l}\text { Estudio } \\
\text { cuasiexperimental }\end{array}$ & + & + & - & + & - & - & - & + & + & + & + & 6 & No \\
\hline Wu et al. ${ }^{26}, 2011$ & $\begin{array}{l}\text { Estudio de } \\
\text { prevalencia }\end{array}$ & + & - & - & + & - & - & - & + & + & + & + & 5 & No \\
\hline Zhang et al. ${ }^{27}, 2015$ & $\begin{array}{l}\text { Estudio prospectivo } \\
\text { de cohorte }\end{array}$ & + & - & - & + & - & + & - & + & + & + & + & 6 & No \\
\hline Zhang et al. ${ }^{28}, 2015$ & $\begin{array}{l}\text { Estudio de } \\
\text { prevalencia }\end{array}$ & + & - & - & + & - & - & - & + & + & + & + & 5 & No \\
\hline Hållmarker et al. ${ }^{29}, 2015$ & Estudio de cohorte & + & + & - & + & - & - & - & + & + & + & + & 6 & No \\
\hline Zhang et al. ${ }^{30}, 2015$ & $\begin{array}{l}\text { Estudio prospectivo } \\
\text { de cohorte }\end{array}$ & + & - & - & + & - & + & - & + & + & + & + & 6 & No \\
\hline Awad et al. ${ }^{31}, 2018$ & Estudio longitudinal & + & - & - & + & + & - & - & + & + & + & + & 6 & No \\
\hline
\end{tabular}

1: criterios de elección; 2: grupos al azar; 3: asignación oculta; 4: grupos similares al inicio; 5 : sujetos cegados; 6: terapeutas cegados; 7: evaluadores cegados; 8: > 85\% resultados de los sujetos iniciales; 9: análisis por «intención de tratar»; 10: comparaciones estadísticas entre grupos; 11: medidas puntuales y de variabilidad.

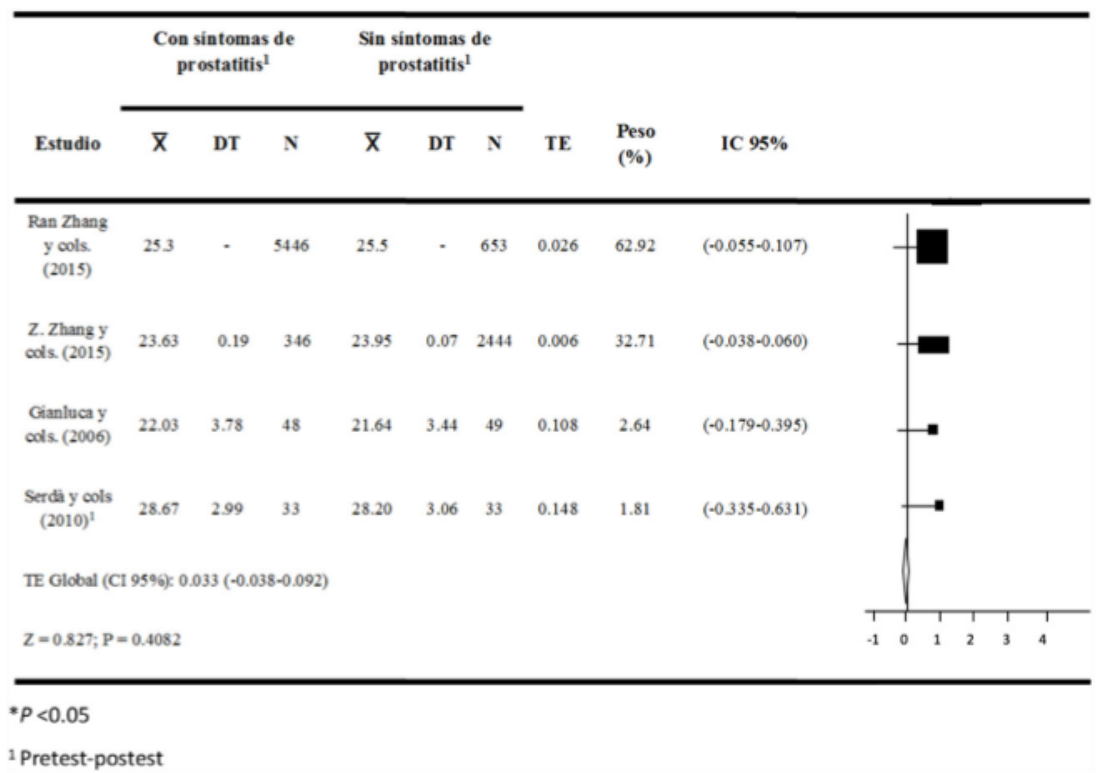

Figura 2 Análisis de la comparación del índice de masa corporal.

global de las intervenciones resultó significativo (TE: 0,11 ; IC $95 \% 0,038$ a 0,$43 ; p=0,024)$.

\section{Discusión}

Según los datos obtenidos tras el análisis de los resultados en nuestra revisión, podemos encuadrar 2 tipos de estudios: aquellos que efectuaron análisis observacionales para comprobar si existía relación entre los síntomas asociados con CP/CPPS y el ejercicio o la actividad física ${ }^{23,26-31}$ y aquellos que realizaron algún programa específico de actividad física para comprobar la relación del mismo con posibles síntomas asociados con CP/CPPS.

En el estudio realizado entre 2002-2004, a 231 hombres de edades comprendidas entre los 20 y los 50 años con síntomas de CP/CPPS positivos se les indicó ejercicio físico moderadamente intenso ${ }^{22}$. Se separaron 2 grupos aleatorizados con 52 integrantes en el grupo de ejercicio aeróbico y 51 integrantes en el grupo placebo/stretching and motion exercises. El papel de la actividad física sobre los sínto- 
Tabla 3 Análisis de los resultados obtenidos de los diferentes artículos seleccionados en la revisión

\begin{tabular}{|c|c|c|c|c|c|c|}
\hline Referencia & Datos demográficos & Tipo de estudio & Duración & $\begin{array}{l}\text { Protocolo de } \\
\text { intervención }\end{array}$ & $\begin{array}{l}\text { Parámetros } \\
\text { medidos }\end{array}$ & Resultados \\
\hline $\begin{array}{l}\text { Giubilei et al. }{ }^{22} \text {, } \\
2007\end{array}$ & $\begin{array}{l}n=231 \\
\text { GE: } 52 \\
\text { GC: } 51 \\
20-50 \text { años }\end{array}$ & $\begin{array}{l}\text { GE: ejercicio aeróbico } \\
\rightarrow \text { AEG } \\
\text { GC: stretching and } \\
\text { motion exercises } \rightarrow \\
\text { P/FlexG }\end{array}$ & 18 semanas & $\begin{array}{l}\text { AEG } \rightarrow \text { ejercicios } \\
\text { aeróbicos } \\
\text { P/FlexG } \rightarrow \text { no } \\
\text { aeróbico. } \\
\text { Actividad física } \\
\text { ligera }\end{array}$ & $\begin{array}{l}\text { NIH-CPSI } \\
\text { Intensidad de } \\
\text { dolor }\end{array}$ & $\begin{array}{l}\text { Los ejercicios aeróbicos } \\
\text { mejoran los síntomas asociados } \\
\text { con CP/CPPS }\end{array}$ \\
\hline $\begin{array}{l}\text { Bartoletti et al. }{ }^{23} \text {, } \\
2009\end{array}$ & $\begin{array}{l}\mathrm{n}=425 \\
\text { G. ciclista: } 245 \\
\text { G. otros deportes: } 105 \\
\text { G. sedentarios (GC): } 75 \\
\text { 38,21 años }\end{array}$ & $\begin{array}{l}\text { Evaluación de los } \\
\text { síntomas genitourinarios } \\
\text { a través del National } \\
\text { Institute of } \\
\text { Health-Chronic } \\
\text { Prostatitis Symptom } \\
\text { Index, the International } \\
\text { Prostatic Symptom Score } \\
\text { y la Pain Visual Analogic } \\
\text { Scale } \\
\text { Evaluación del dolor } \\
\text { perianal y zona } \\
\text { suprapúbica }\end{array}$ & No mostrados & $\begin{array}{l}\text { Recogida de datos } \\
\text { observacionales }\end{array}$ & $\begin{array}{l}\text { NIH-CPSI } \\
\text { IPS3Score } \\
\text { EVA } \\
\text { PSA Ag }\end{array}$ & $\begin{array}{l}\text { No hay diferencias } \\
\text { significativas entre deportistas } \\
\text { y GC } \\
\text { EVA con diferencias } \\
\text { significativas entre grupos. Los } \\
\text { ciclistas dan puntuaciones más } \\
\text { altas en la zona perianal y } \\
\text { suprapúbica }\end{array}$ \\
\hline $\begin{array}{l}\text { Serdà et al. }{ }^{24} \text {, } \\
2010\end{array}$ & $n=33$ & $\begin{array}{l}\text { Programa progresivo de } \\
\text { fuerza-resistencia para } \\
\text { pacientes con cáncer de } \\
\text { próstata }\end{array}$ & 24 semanas & $\begin{array}{l}16 \text { semanas } \\
\text { control directo } \\
8 \text { semanas trabajo } \\
\text { autónomo }\end{array}$ & $\begin{array}{l}\text { Antropometría, } \\
\text { fuerza/resistencia, } \\
\text { TA, dolor, calidad } \\
\text { de vida }\end{array}$ & $\begin{array}{l}\text { Mejora en extremidades } \\
\text { inferiores en mayor medida } \\
\text { que en el tronco. Mejora en } \\
\text { calidad de vida. Disminución } \\
\text { de riesgo cardiovascular. } \\
\text { Disminución de HTA }\end{array}$ \\
\hline $\begin{array}{l}\text { Serdà et al. }{ }^{25}, \\
2013\end{array}$ & $n=33$ & $\begin{array}{l}\text { Programa progresivo de } \\
\text { fuerza-resistencia para } \\
\text { pacientes con cáncer de } \\
\text { próstata }\end{array}$ & 24 semanas & $\begin{array}{l}16 \text { semanas } \\
\text { control directo } \\
8 \text { semanas trabajo } \\
\text { autónomo }\end{array}$ & $\begin{array}{l}\text { Antropometría, } \\
\text { fuerza/resistencia, } \\
\text { TA, dolor, calidad } \\
\text { de vida }\end{array}$ & $\begin{array}{l}\text { Mejora en extremidades } \\
\text { inferiores en mayor medida } \\
\text { que en el tronco. Mejora en } \\
\text { calidad de vida. Disminución } \\
\text { de riesgo cardiovascular. } \\
\text { Disminución de HTA }\end{array}$ \\
\hline Wu et al. ${ }^{26}, 2011$ & $\begin{array}{l}n=1.868 \\
19.78 \text { años }\end{array}$ & $\begin{array}{l}4.930 \text { no prostatitis } \\
4.820 \text { prostatitis }\end{array}$ & 3 meses & $\begin{array}{l}\text { Recogida de datos } \\
\text { observacionales }\end{array}$ & $\begin{array}{l}\text { NIH-IV prostatitis } \\
\text { en relación con } \\
\text { medidas } \\
\text { antropométricas, } \\
\text { consumo de } \\
\text { tabaco, HTA y } \\
\text { nivel de colesterol }\end{array}$ & $\begin{array}{l}\text { No hay diferencias } \\
\text { significativas entre grupos al } \\
\text { analizar los parámetros } \\
\text { estudiados }\end{array}$ \\
\hline $\begin{array}{l}\text { Zhang et al. }{ }^{27} \text {, } \\
2015\end{array}$ & $\mathrm{n}=20.918$ sanos & $\begin{array}{l}\text { Comprobación de nivel } \\
\text { de actividad física }\end{array}$ & 24 años & $\begin{array}{l}\text { Actividad física de } \\
\text { ocio }\end{array}$ & $\begin{array}{l}\text { MET* }^{*}{ }^{*} w^{-1} \\
\text { Sintomas CP/CPPS }\end{array}$ & $\begin{array}{l}689 \text { de los } 20.918 \text { hombres } \\
\text { desarrollaron síntomas de } \\
\text { CP/CPPS }\end{array}$ \\
\hline
\end{tabular}


Tabla 3 (continuación)

\begin{tabular}{|c|c|c|c|c|c|c|}
\hline Referencia & Datos demográficos & Tipo de estudio & Duración & $\begin{array}{l}\text { Protocolo de } \\
\text { intervención }\end{array}$ & $\begin{array}{l}\text { Parámetros } \\
\text { medidos }\end{array}$ & Resultados \\
\hline $\begin{array}{l}\text { Zhang et al. }{ }^{28} \text {, } \\
2015\end{array}$ & $\begin{array}{l}\mathrm{n}=2.790 \\
20-84 \text { años } \\
\text { China }\end{array}$ & $\begin{array}{l}34,20 \% \text { prostatitis } \\
40,30 \% \text { no prostatitis }\end{array}$ & 4 meses & $\begin{array}{l}\text { NIH-CPSI } \\
\text { FAMHES }\end{array}$ & $\begin{array}{l}\text { Sintomas de } \\
\text { prostatitis }\end{array}$ & $\begin{array}{l}\text { Fumar, beber, ausencia de } \\
\text { actividad física y elevado nivel } \\
\text { de glucosa en plasma se } \\
\text { asocian con un incremento en } \\
\text { el riesgo de síntomas } \\
\text { prostáticos. No hay relación } \\
\text { con la edad }\end{array}$ \\
\hline $\begin{array}{l}\text { Hållmarker } \\
\text { et al. }{ }^{29}, 2015\end{array}$ & $\begin{array}{l}n=185.412 \text { esquiadores } \\
n=184.617 \text { población } \\
\text { general }\end{array}$ & $\begin{array}{l}\text { Incidencia de número de } \\
\text { casos a lo largo del } \\
\text { tiempo }\end{array}$ & $1989-2010$ & $\begin{array}{l}\text { Registro de } \\
\text { esquiadores a } \\
\text { través del número } \\
\text { de registro } \\
\text { nacional sueco. } \\
\text { Registro de no } \\
\text { esquiadores a } \\
\text { través del registro } \\
\text { oficial de la } \\
\text { población sueca }\end{array}$ & $\begin{array}{l}\text { Número de casos } \\
\text { Número de casos } \\
\text { de cáncer por } \\
1.000 \text { personas al } \\
\text { año }\end{array}$ & $\begin{array}{l}\text { Disminución de un } 6 \% \text { en el } \\
\text { riesgo de cáncer invasivo es } \\
\text { esquiadores frente a no } \\
\text { esquiadores }(1.827 \text { vs. } 1.435) \\
(1,51 \text { vs. } 1,22 ; p>0,001) \\
\text { La probabilidad de incrementar } \\
\text { el número de casos de cáncer } \\
\text { de próstata en } 20 \text { años supone } \\
\text { un } 4,6 \% \text { más en esquiadores } \\
\text { frente a un } 3,8 \% \text { en no } \\
\text { esquiadores }\end{array}$ \\
\hline $\begin{array}{l}\text { Zhang et al. }{ }^{30} \text {, } \\
2015\end{array}$ & $\begin{array}{l}n=19.791 \\
50,7 \text { años }\end{array}$ & $\begin{array}{l}\text { Evaluación de los } \\
\text { sintomas CP/CPPS a } \\
\text { través del cuestionario } \\
\text { del National Institute of } \\
\text { Health-Chronic } \\
\text { Prostatitis Symptom } \\
\text { Index, en relación con } \\
\text { información médica y } \\
\text { factores de vida }\end{array}$ & 22 años & $\begin{array}{l}\text { Cuestionarios } \\
\text { validados, así } \\
\text { como historial de } \\
\text { enfermedades } \\
\text { uretrales. Estudio } \\
\text { de cohortes }\end{array}$ & $\begin{array}{l}\text { MET* }^{*}{ }^{*} \mathrm{wk}^{-1} \\
\text { Síntomas CP/CPPS } \\
\text { NIH-CPSI } \\
\text { intensidad de } \\
\text { dolor }\end{array}$ & $\begin{array}{l}653 \text { casos con CP/CPPS. No } \\
\text { asociación con obesidad, fumar } \\
\text { o hipertensión y desarrollo de } \\
\text { síntomas CP/CPPS }\end{array}$ \\
\hline $\begin{array}{l}\text { Awad et al. }{ }^{31}, \\
2018\end{array}$ & $\begin{array}{l}n=3.932 \\
\text { G. ciclista baja } \\
\text { intensidad: } 1.858 \\
\text { G. ciclista alta } \\
\text { intensidad: } 916 \\
\text { G. natación/corredores: } \\
1.158 \\
1.361 \text { practicaban } \\
\text { ciclismo y natación } 0 \\
\text { carrera }\end{array}$ & $\begin{array}{l}\text { Evaluación de la relación } \\
\text { de síntomas urinarios y } \\
\text { sexuales por la } \\
\text { realización de ciclismo }\end{array}$ & 9 meses & Diseño transversal & $\begin{array}{l}\text { Síntomas CP/CPPS } \\
\text { NIH-CPSI } \\
\text { intensidad de } \\
\text { dolor }\end{array}$ & $\begin{array}{l}\text { No hay relación entre ciclismo } \\
\text { y mayores problemas urinarios } \\
\text { o sexuales comparados con } \\
\text { nadadores o corredores. Los } \\
\text { ciclistas presentan tamaños } \\
\text { estructurales de la uretra } \\
\text { mayores que el resto de los } \\
\text { deportistas }\end{array}$ \\
\hline
\end{tabular}

AEG: aerobic exercise group; CP/CPPS: prostatitis crónica/síndrome de dolor pélvico crónico; EVA: escala visual analógica; G: grupo; CG: grupo control; GE: grupo experimental; HTA: hipertensión arterial; NIH-CPSI: National Institute of Health-Chronic Prostatitis Symptom Index; P/FlexG: placebo/streching and motion exercises group; TA: tensión arterial. 


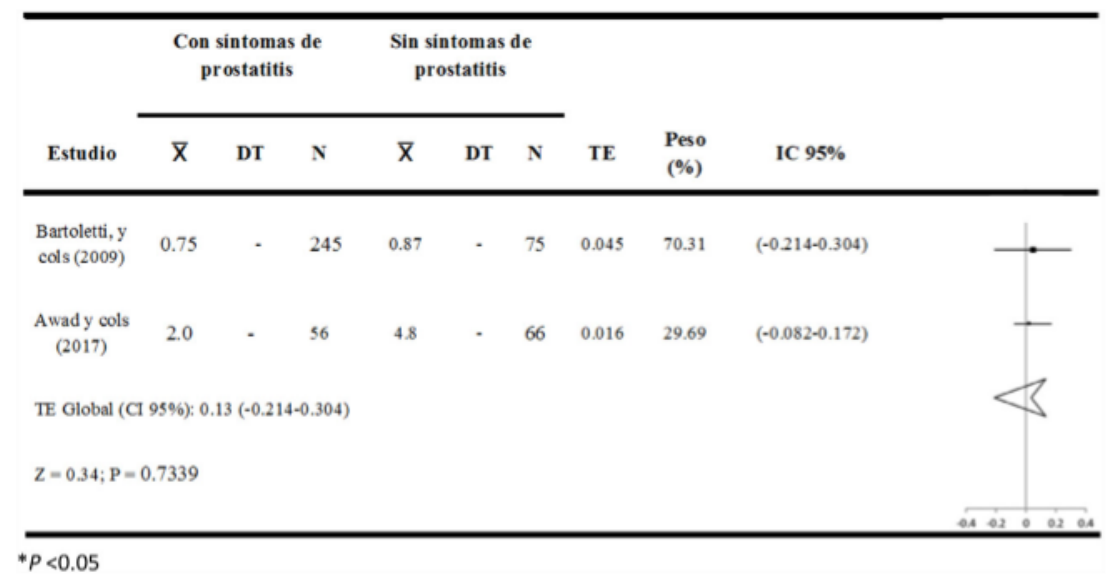

Figura 3 Análisis de la comparación del índice de masa corporal (calidad de vida).

\begin{tabular}{|c|c|c|c|c|c|c|c|c|c|c|}
\hline \multirow[b]{2}{*}{ Estudio } & \multicolumn{3}{|c|}{$\begin{array}{l}\text { Con sintomas de } \\
\text { prostatitis' }^{1}\end{array}$} & \multicolumn{3}{|c|}{$\begin{array}{c}\text { Sin sintomas de } \\
\text { prostatitis' }\end{array}$} & \multirow[b]{2}{*}{ TE } & \multirow[b]{2}{*}{$\begin{array}{l}\text { Peso } \\
(\%)\end{array}$} & \multirow[b]{2}{*}{ IC 95\% } & \\
\hline & X & DT & $\mathbf{N}$ & 又 & DT & $\mathbf{N}$ & & & & \\
\hline $\begin{array}{l}\text { Bartoletti y } \\
\text { cols (2009) }\end{array}$ & 2.20 & $\cdot$ & 245 & 1.28 & $\cdot$ & 75 & 0.034 & 57.41 & $(-0.214-0.304)$ & \\
\hline $\begin{array}{l}\text { Chunlei Wu } \\
\text { y cols } \\
\text { (2013) }\end{array}$ & 1,0 & - & 48 & 1.7 & $\cdot$ & 49 & 0.052 & 24.23 & $(-0.346-0.450)$ & \\
\hline $\begin{array}{l}\text { Serdà y cols } \\
\qquad(2010)^{1}\end{array}$ & 3.79 & 2.54 & 33 & 1.03 & 0.92 & 33 & 1.42 & 13.10 & $(0.882-1965)^{*}$ & $\rightarrow$ \\
\hline TE Global (Cl & $95 \%): 0$ & $1(0.03$ & $.43)^{*}$ & & & & & & & $B$ \\
\hline $\mathrm{Z}=2.248 ; \mathrm{P}=$ & 0.0246 & & & & & & & & & 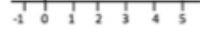 \\
\hline
\end{tabular}

Figura 4 Análisis de la comparación de la incontinencia urinaria.

mas de la CP/CPPS se analizó por medio de un estudio prospectivo, aleatorizado a doble ciego, en el cual se observó que, a pesar de que inicialmente casi la mitad de los pacientes sometidos a ejercicio aeróbico presentaron una exacerbación del dolor, la cohorte de pacientes que realizaban un ejercicio aeróbico mejoraban significativamente en la puntuación total del cuestionario NIH-CPSI y su calidad de vida respecto al grupo placebo a las 18 semanas. Se obtuvo como conclusión que el ejercicio aeróbico representa una opción válida de tratamiento para la CP/CPPS.

Serdà et al. llevaron a cabo 2 estudios; ambos se engloban dentro de unos estudios cuasiexperimentales de 33 participantes en fase de tratamiento del cáncer de próstata. Las variables estudiadas fueron: variables antropométricas, fuerza-resistencia, tensión arterial, fatiga, incontinencia, dolor y calidad de vida. Dichas variables se estudiaron durante 24 semanas, observándose una mejora significativa de la capacidad de la fuerza-resistencia, más evidente en las extremidades inferiores, así como una mejora en la tensión arterial, la incontinencia urinaria y el dolor ${ }^{24,25}$.

Por el contrario, en el estudio observacional analítico de cohorte desarrollado por Wu et al. para evaluar los síntomas genitourinarios en sujetos que practican ciclismo comparado con otros deportes o con gente sedentaria (grupo control) ${ }^{26}$, evalúan a 425 hombres de 38,21 años de edad media, los cuales fueron divididos en 3 grupos: 245 ciclistas, 105 otros deportes, 75 sedentarios. No existen diferencias significativas entre deportistas y el grupo control. En la escala EVA de dolor hubo diferencias significativas, dando puntuaciones más altas los ciclistas en las zonas perianal y suprapúbica. No hubo diferencias entre grupos en síntomas urinarios o en el antígeno específico prostático.

Diversos estudios demuestran que la edad, el tabaquismo y un nivel educativo bajo se relacionan con un incremento de la prostatitis asintomática (NIH-IV) ${ }^{27}$. Otros autores, por otra parte, indican que el tabaquismo, el consumo de alcohol, una baja actividad física y una glucosa elevada en sangre se relacionan con un elevado riesgo de prostatitis y con la disfunción eréctil ${ }^{28}$.

En el estudio de cohorte sobre factores de vida relacionados con CP/CPPS en profesionales sanitarios con un tamaño de muestra de $\mathbf{5 1 . 5 2 9}$ hombres de edades comprendidas entre los 40 y los 75 años, realizado entre los años 1986 y 2008 , se demuestra que no existe relación entre factores 
de riesgo como obesidad, tabaquismo e hipertensión con la prostatitis crónica. Asimismo, dicho trabajo indica que no existe asociación con el índice de masa corporal, la circunferencia de la cintura, el índice cintura-cadera, el consumo de tabaco y la hipertensión con el riesgo de CP/CPPS ${ }^{30}$.

Un estudio de cohorte llevado a cabo durante los años 1989-2010 con 185.412 esquiadores de larga distancia y no participantes en la carrera de larga distancia de esquí Vasaloppet, realizada en Suecia durante los años mendionados ${ }^{29}$, estimó las ratios de peligrosidad y probabilidad de padecer cáncer durante los más de 20 años de seguimiento, comparando aquellos sujetos que participaron en la mencionada carrera de esquí de fondo con aquellos que nunca realizaron dicha carrera dentro de la población sueca. Los resultados que obtuvieron reflejan una disminución del $6 \%$ en el riesgo de cáncer invasivo, así como una reducción del $32 \%$ en cánceres relacionados con el consumo de tabaco, el peso corporal, la actividad física habitual o el consumo de frutas y verduras como factores etiológicos.

En este estudio se pone de manifiesto que los ciclistas no tienen más problemas o disfunciones sexuales y urinarias que los nadadores y los corredores. Sin embargo, los ciclistas son más propensos a las estenosis uretrales; a más tiempo montando en bicicleta y a mayor manillar, aumenta la asociación, con bajas posibilidades de llagas genitales y entumecimiento ${ }^{31}$.

\section{Conclusiones}

La variabilidad asociada a los diseños experimentales incluidos en el metaanálisis representa una heterogeneidad en los efectos de los distintos programas o intervenciones de actividad física para el tratamiento de la prostatitis crónica. Dicha variabilidad podría explicar el hallazgo de TE pequeños para las distintas variables dependientes analizadas en nuestro estudio, si bien la literatura incluida en el metaanálisis parece verosímil en torno a la eficacia de los tratamientos. Los TE obtenidos apuntan a que la efectividad de los programas de tratamiento alternativos, empleando vehicularmente la actividad física, puede guardar una relación con el tipo de intervención realizada. Hallar los mecanismos de respuesta y tratamiento siguiendo un diseño metodológico homogéneo puede ser de gran utilidad para la realización de intervenciones terapéuticas más eficaces en el futuro.

\section{Responsabilidades éticas}

Protección de personas y animales. Los autores declaran que para esta investigación no se han realizado experimentos en seres humanos ni en animales.

Confidencialidad de los datos. Los autores declaran que han seguido los protocolos de su centro de trabajo sobre la publicación de datos de pacientes.

Derecho a la privacidad y consentimiento informado. Los autores declaran que en este artículo no aparecen datos de pacientes.

\section{Conflicto de intereses}

Los autores declaran no tener ningún conflicto de intereses en relación con la elaboración de este manuscrito y que no hemos recibido financiación para su elaboración.

\section{Agradecimientos}

El presente artículo ha formado parte de la tesis doctoral del autor Víctor Jiménez Díaz-Benito. El autor agradece al equipo de investigación su implicación en la realización de esta investigación.

\section{Bibliografía}

1. Irwin DE, Milsom I, Kopp Z, Abrams P, Artibani W, Herschorn S. Prevalence, severity, and symptom bother of lower urinary tract symptoms among men in the EPIC study: Impact of overactive bladder. Eur Urol. 2009;56:14-20.

2. Vicari E, La Vignera S, Castiglione R, Condorelli RA, Vicari LO, Calogero AE. Chronic bacterial prostatitis and irritable bowel syndrome: Effectiveness of treatment with rifaximin followed by the probiotic VSL\#3. Asian J Androl. 2014;16:735-9.

3. Jiménez-Pacheco A, Jiménez-Pacheco A, Roldán-López M. Therapeutic strategies for the treatment of chronic prostatitis/chronic pelvic pain syndrome. Rev Int Androl. 2016;14:19-26.

4. Heras-Cañas V, Gutiérrez-Soto B, Serrano-García ML, VázquezAlonso F, Navarro-Marí JM, Gutiérrez-Fernández J. [Chronic bacterial prostatitis. Clinical and microbiological study of 332 cases] Spanish. Med Clin (Barc). 2016;147:144-7.

5. Litwin MS, McNaughton-Collins M, Fowler FJ Jr, Nickel JC, Calhoun EA, Pontari MA, et al. The National Institutes of Health chronic prostatitis symptom index: Development and validation of a new outcome measure. Chronic Prostatitis Collaborative Research Network. J Urol. 1999;162:369-75.

6. Kai B, Lee KD, Andrews G, Wilkinson M, Forster BB. Puck to pubalgia: Imaging of groin pain in professional hockey players. Can Assoc Radiol J. 2010;61:74-9.

7. St Sauver JL, Jacobsen SJ, Jacobson DJ, McGree ME, Girman $\mathrm{CJ}$, Nehra A, et al. Statin use and decreased risk of benign prostatic enlargement and lower urinary tract symptoms. BJU Int. 2011;107:443-50.

8. Robertson BA, Barker PJ, Fahrer M, Schache AG. The anatomy of the pubic region revisited: Implications for the pathogenesis and clinical management of chronic groin pain in athletes. Sports Med. 2009;39:225-34.

9. Asplund C, Barkdull T, Weiss BD. Genitourinary problems in bicyclists. Curr Sports Med Rep. 2007;6:333-9.

10. Leibovitch I, Mor Y. The vicious cycling: Bicycling related urogenital disorders. Eur Urol. 2005;47:277-86, discussion 286-7.

11. Estabrooks PA, Glasgow RE, Dzewaltowski DA. Physical activity promotion trough primary care. JAMA. 2003;289: 2913-6.

12. Pate RR, Pratt M, Blair SN, Haskell WL, Macera CA, Bouchard $\mathrm{C}$, et al. Physical activity and public health: A recommendation from the Centers for Disease Control and Prevention and the American College of Sport Medicine. JAMA. 1995;273:402-7.

13. Liberati A, Altman DG, Tetzlaff J, Mulrow C, Gøtzsche PC, loannidis JP, et al. The PRISMA statement for reporting systematic reviews and meta-analyses of studies that evaluate healthcare interventions: Explanation and elaboration. Ann Intern Med. 2009;151:W-65-94. 
14. De Morton NA. The PEDro scale is a valid measure of the methodological quality of clinical trials: A demographic study. Aust J Physiother. 2009;55:129-33.

15. Maher CG, Sherrington C, Herbert RD, Moseley AM, Elkins M. Reliability of the PEDro scale for rating quality of randomized controlled trials. Phys Ther. 2003;83:713-21.

16. Wright JG, Swiontkowski MF, Heckman JD. Introducing levels of evidence to the journal. J Bone Joint Surg Am. 2003;85-A:1-3.

17. Hedges LV. Estimation of effect size from a series of independent experiments. Psychol Bull. 1982;92:490-9.

18. Lipsey MW, Wilson DB. Practical meta-analysis. Thousand Oaks, CA: Sage Publications, Inc.; 2001.

19. Hedges LV, Olkin I. Statistical methods for meta-analysis. New York: Academic Press; 1985.

20. Cohen J. Statistical power analysis for the behavioral sciences. 2 nd Hillsdale, NJ: Lawrence Erlbaum Associates, Publishers; 1988.

21. Moseley AM, Herbert RD, Sherrington C, Maher CG. Evidence for physiotherapy practice: A survey of the Physiotherapy Evidence Database (PEDro). Aust J Physiother. 2002;48:43-9.

22. Giubilei G, Mondaini N, Minervini A, Saieva C, Lapini A, Serni S, et al. Physical activity of men with chronic prostatitis/chronic pelvic pain syndrome not satisfied with conventional treatments-Could it represent a valid option? The physical activity and male pelvic pain trial: A double-blind, randomized study. J Urol. 2007;177:159-65.

23. Bartoletti R, Cai T, Mondaini N, Giubilei G, Gavazzi A, Mazzoli S, et al. Cycling and genitourinary symptoms: Results from an observational analytical cohort study. J Androl Sci. 2009;16:98-103.
24. Serdà BC, Monreal P, del Valle A. Physical exercise as complementary treatment in prostate cancer. Apunts Med Esport. 2010;45:81-93.

25. Wu C, Zhang Z, Lu Z, Liao M, Zhang Y, Xie Y, et al. Prevalence of and risk factors for asymptomatic inflammatory (NIH-IV) prostatitis in Chinese men. PLoS One. 2013;8:e71298.

26. Serdà BC, Monreal P, del Valle A. Quality of life and prostate cancer: A progressive resistance strength program. Rev Int Med Cienc Act Fis Dep. 2011;11:362-83.

27. Zhang R, Chomistek AK, Dimitrakoff JD, Giovannucci EL, Willett WC, Rosner BA, et al. Physical activity and chronic prostatitis/chronic pelvic pain syndrome. Med Sci Sports Exerc. 2015 Apr;47:757-64.

28. Zhang Z, Li Z, Yu Q, Wu C, Lu Z, Zhu F, et al. The prevalence of and risk factors for prostatitis-like symptoms and its relation to erectile dysfunction in Chinese men. Andrology. 2015;3:1119-24.

29. Hållmarker U, James S, Michaëlsson K, Ärnlöv J, Sandin F, Holmberg L. Cancer incidence in participants in a long-distance ski race (Vasaloppet Sweden) compared to the background population. Eur J Cancer. 2015;51:558-68.

30. Zhang R, Sutcliffe S, Giovannucci E, Willett WC, Platz EA, Rosner BA, et al. Lifestyle and risk of chronic prostatitis/chronic pelvic pain syndrome in a cohort of United States male health professionals. J Urol. 2015;194:1295-300.

31. Awad MA, Gaither TW, Murphy GP, Chumnarnsongkhroh T, Metzler I, Sanford T, et al. Cycling, and male sexual and urinary function: Results from a large, multinational, cross-sectional study. J Urol. 2018;199:798-804. 